\title{
The immediate and delayed effects of different types of vagotomy on human gastric myoelectrical activity ${ }^{1}$
}

\author{
C. J. STODDARD, R. SMALLWOOD, B. H. BROWN, AND H. L. DUTHIE \\ From the University Surgical Unit, Sheffield Royal Infirmary
}

SUMMARY The immediate and delayed effects of different types of vagotomy on the antral myoelectrical activity have been studied in 52 patients who had previously undergone either highly selective vagotomy (HSV) or truncal vagotomy and pyloroplasty (TV + P) for the treatment of chronic duodenal ulceration up to eight years previously. The frequency of the electrical activity was not statistically different between the two types of operation. After HSV the triphasic waveshape of the antral myoelectrical activity was retained; it was recorded more frequently in the delayed tests $(98.0 \pm 1.7 \%)$ of the recording period than in immediate tests $(74.7 \pm 6.5 \%)$ whereas mean amplitude in the immediate tests $(1.59 \pm 0.13 \mathrm{mV})$ was not significantly changed in the delayed tests $(1.49 \pm 0.08 \mathrm{mV})$. After TV $+\mathrm{P}$ the waveform was more sinusoidal in shape, being recorded more often in the delayed tests $(91.7 \pm 2.7 \%)$ than in the immediate tests $(41.4 \pm 8.9 \%)$ although the mean amplitude between the immediate $(0.90 \pm 0.06 \mathrm{mV})$ and delayed tests $(0.94 \pm 0.07 \mathrm{mV})$ was not significantly different. The only significant change in the myoelectrical activity with the passage of time after vagotomy was an increase in the percentage activity.

Soon after vagotomy was introduced for the treatment of duodenal ulcer (Dragstedt and Owens, 1943) it became apparent that vagal section impaired gastric motility (Storer, Thornton, and Dragstedt, 1945). Since myoelectrical activity is directly related to motor activity changes in the pacesetter potential can be expected following vagotomy. Studies performed intraoperatively show no change in the myoelectrical activity (Nagaoka, 1968; McIntyre, Deitel, Baida, and Jalil, 1969) although changes are apparent in the immediate postoperative period in both man (Morton, 1954; Kwong, Brown, Whittaker, and Duthie, 1970) and in the canine stomach (Nelsen, Eigenbrodt, Keoshian, Bunker, and Johnson, 1967; Kelly and Code, 1969; Stoddard, Brown, and Duthie, 1973a). These alterations in antral myoelectrical activity are related to the extent of gastric vagal denervation (Stoddard, Waterfall, Brown, and Duthie, 1973b). Persisting irregular activity occurs in the canine stomach many weeks after TV (Nelsen et $a l, 1967)$ and long-term changes in human myoelectrical activity after vagotomy may therefore be expected. In the present study patients after different types of vagotomy have had immediate

${ }^{1}$ Based in part on a communication to the Surgical Research Society, July 1974.

Received for publication 18 December 1974. postoperative or delayed studies of antral myoelectrical activity performed to determine whether the changes are transient or not.

\section{Methods}

Fifty-two subjects (47 male and five female) were studied up to eight years after HSV or TV $+P$ for chronic duodenal ulceration (table I). Informed consent was obtained for all investigations.

The studies were performed with the patients lying down after fasting overnight. A modified nasogastric tube was passed into the gastric antrum under radiographic control and attached to the antral mucosa by suction at a negative pressure of $20 \mathrm{~cm}$ of mercury, which was maintained for the duration of the test. The tube, identical to that previously used in this department (Kwong, Brown, Whittaker, and Duthie, 1972), bore a stainless steel electrode $1 \mathrm{~cm}$ from its tip from which monopolar

\begin{tabular}{llll}
\hline & Immediate & Delayed & Totals \\
\hline HSV & 9 & 10 & 19 \\
TV + P & 14 & 19 & 33 \\
Totals & 23 & 29 & \\
\hline
\end{tabular}

Table 1 The number of patients studied after each type of vagotomy at each recording stage 
recordings of the antral myoelectrical activity were made. The indifferent electrode was a metal disc applied to the scarified skin of the right iliac fossa. Signals from the electrode were fed into an AC amplifier and displayed on a UV recorder. The frequency response of this system was within $\pm 3 \mathrm{~dB}$ from $0.016 \mathrm{~Hz}$ to $2.0 \mathrm{KHz}$. A parallel output was fed into a filter with frequency response from 1 to 6 cycles per minute $( \pm 3 \mathrm{~dB}$ from $0.016 \mathrm{~Hz}$ to $0.1 \mathrm{~Hz}$ ). Synchronous recording of gastric motility was made by the open-tipped catheter technique using a pressure transducer (SE 76/8). An attached second tube, with its first opening $6 \mathrm{~cm}$ proximal to the electrode, was used to aspirate continuously the gastric secretions.

Recording was commenced 30 minutes after intubation during which time the patients became accustomed to the tube and residual gastric contents were aspirated. All 52 patients underwent a control 60-minute study which was followed in 32 patients by an intravenous injection of soluble insulin, $0 \cdot 2$ iu per kg body weight, and a further two-hour recording. Of the remaining 20 patients, 17 had had their gastric response to insulin measured at a separate study. All gastric secretory responses were negative as judged by Hollander's criteria (1948), except for the following:

\begin{tabular}{llll}
\hline HSV (immediate) & $\ldots$ & $\ldots$ & 1 late positive \\
TV + P (immediate) & $\ldots$ & 2 late positives \\
HSV (delayed) & $\ldots$ & $\ldots$ & 2 late positives \\
TV + P (delayed) & $\ldots$ & $\ldots$ & 3 late positives and 1 early positive
\end{tabular}

Twenty-one patients had previously undergone a prevagotomy control recording without the injection of any pharmacological agents.

The recordings were analysed as follows. The configuration and rhythm of the main waveform known as the pacesetter potential (PSP) were noted for each patient and the percentage time that regular activity was present calculated from the total recording time. The mean amplitude of the PSP for each patient was determined by summing the amplitude of each individual cycle and dividing by the total number of cycles. The frequency was calculated by counting the number of cycles during each minute of recording and expressing the result as mean frequency in cycles per minute. Statistical analyses were performed using Student's $t$ test. Results are expressed \pm 1 SEM.

\section{Results}

ELECTRICAL ACTIVITY

Before vagotomy

The 21 patients tested before vagotomy all had regular antral myoelectrical activity which was recorded for $96.1 \pm 1.6 \%$ of the recording period. The normal antral triphasic waveshape was always present with an amplitude of $2.04 \pm 0.11 \mathrm{mV}$ (fig 1). The mean frequency of this electrical activity was $3.07 \pm 0.04 \mathrm{c} / \mathrm{m}$ and in no patient did it vary more than $\pm 0.15 \mathrm{c} / \mathrm{m}$. These results are similar to previous measurements of antral myoelectrical activity in preoperative patients recorded in this department (Duthie, Kwong, Brown, and Whittaker, 1971; Stoddard et al, 1973b).

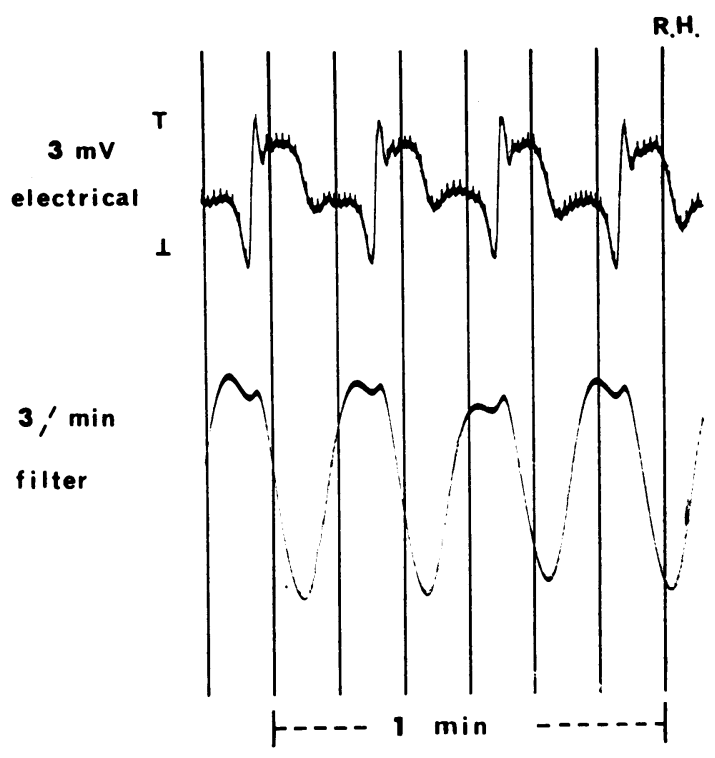

Fig 1 Preoperative recording of electrical activity from the gastric antrum showing the normal triphasic pacesetter potential with a frequency of $3 \cdot 1$ cycles per minute and amplitude of $2.85 \mathrm{mV}$.

\section{After vagotomy}

Two patients, one following HSV and one following $T V+P$, had irregular activity present for the entire control recording period and their studies are considered separately in greater detail below. Of the

\begin{tabular}{lll}
\hline & \multicolumn{2}{l}{ Postoperative } \\
\cline { 2 - 3 } & Immediate & Delayed \\
\hline Preoperative & $3.07 \pm 0.04$ & \\
HSV & $3.18 \pm 0.06$ & $3.08 \pm 0.11$ \\
TV + P & $3.06 \pm 0.05$ & $3.13 \pm 0.05$ \\
\hline
\end{tabular}

Table II The mean frequency of the PSP before and after each type of vagotomy 


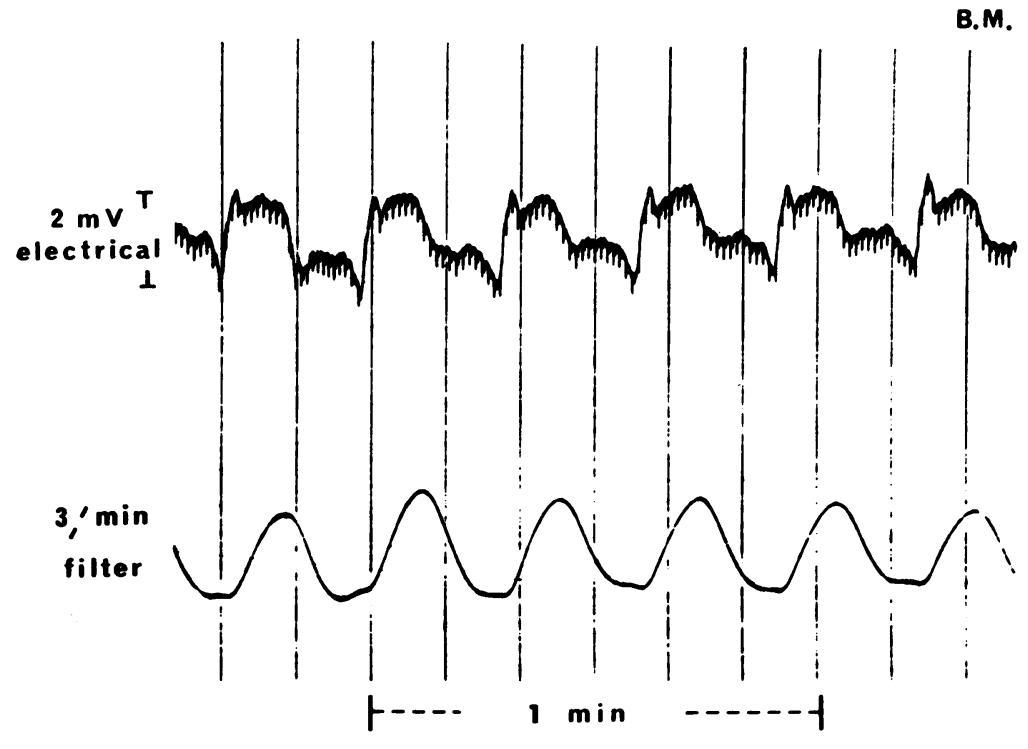

Fig 2 Recording of electrical activity from the gastric antrum one year after highly selective vagotomy showing regular triphasic pacesetter potentials throughout the record with an amplitude of $2 \mathrm{mV}$.

remaining 50 patients there was no significant difference in mean frequency of electrical activity between those patients who had received either type of vagotomy nor between the immediate and delayed tests. The postvagotomy frequencies were not significantly different from the preoperative result (table II).

Following HSV the triphasic waveshape of the PSP was retained in all patients (fig 2). The mean amplitude of the PSP was reduced after HSV in all patients but this reduction was not statistically different between the immediate, $1.59 \pm 0.13 \mathrm{mV}$, and the delayed tests, $1.49 \pm 0.08 \mathrm{mV}$. Regular electrical activity was recorded for $74.7 \pm 6.5 \%$ of the recording period in the immediate postoperative tests and in the delayed studies was present for $98.0 \pm 1.7 \%$ of the time, being present for the entire recording period in seven of the 10 patients studied.

The triphasic waveshape becomes more sinusoidal in character after TV (fig 3) and this alteration was present in the immediate postoperative studies and persisted in all the delayed tests. A larger reduction in mean amplitude of the PSP occurred after TV $+\mathbf{P}$ than HSV although there was no significant difference between the immediate $(0.90 \pm 0.06 \mathrm{mV})$ and delayed tests $(0.94 \pm 0.07 \mathrm{mV})$. Regular electrical activity was present less frequently in the immediate postoperative studies $(41.4 \pm 8.9 \%)$ than in delayed tests $(91 \cdot 7 \pm 2 \cdot 7 \%)$.

Comparison of results in the immediate and delayed tests shows that the decrease in amplitude after both types of vagotomy and alteration in

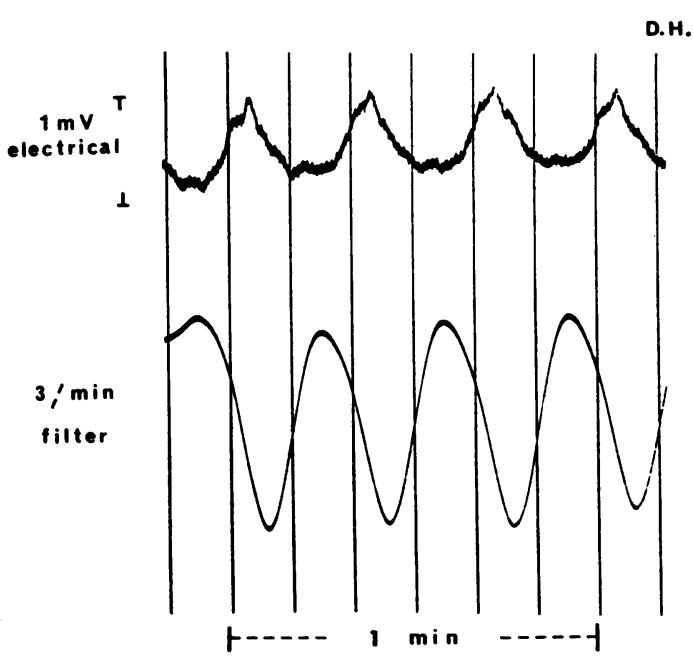

Fig 3 Recording of electrical activity from the gastric antrum one year after truncal vagotomy and pyloroplasty showing regular pacesetter potentials with a more sinusoidal wave shape and amplitude of only $0.9 \mathrm{mV}$.

waveshape after TV $+\mathbf{P}$ are both permanent changes and related to the extent of the gastric vagal denervation. There is a statistically significant difference in the amplitude $(P<0.05)$ between the two types of operation at both recording periods (fig 4) although for each kind of vagotomy there is no difference between the immediate and delayed tests. 


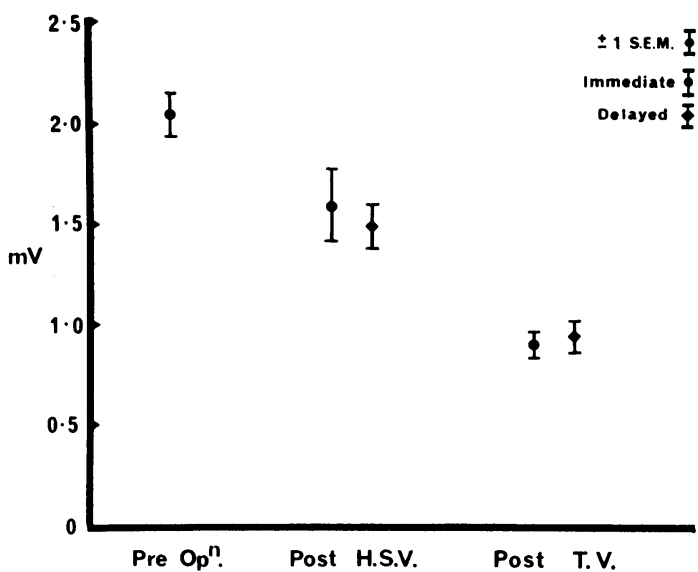

Fig 4 The mean amplitude of the pacesetter potential during the 60-minute control period in preoperative patients and following the two types of vagotomy (HSV, highly selective vagotomy, and $T V$, truncal vagotomy). Following each type of vagotomy the mean amplitude for the immediate and delayed tests are shown.

There is, however, a difference in the percentage time that regular myoelectrical activity can be recorded between the immediate and delayed studies. In the delayed studies activity is present for almost the entire recording period following both types of vagotomy and statistically neither is different from the prevagotomy figures (fig 5).

\section{IRREGULAR ELECTRICAL ACTIVITY}

One patient after each type of operation had irregular activity present throughout the control recording

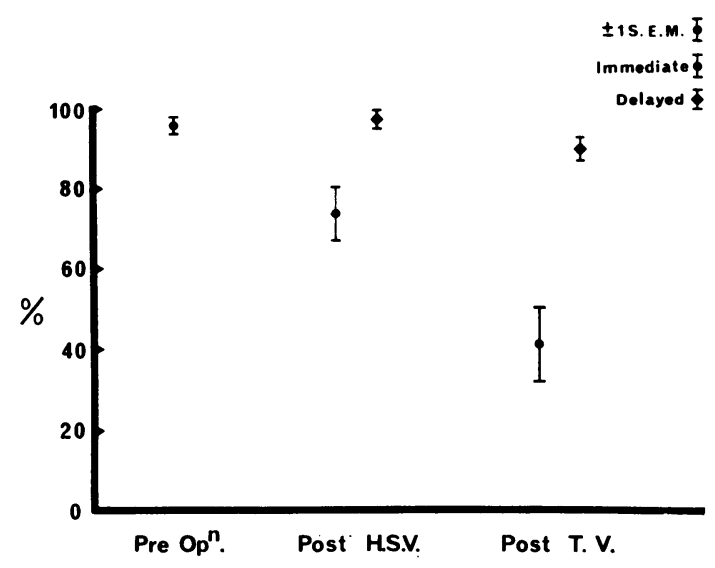

Fig 5 The mean percentage time during which regular myoelectrical activity was present during the 60-minute control period in preoperative patients and following highly selective and truncal vagotomies. Following either type of vagotomy regular activity is seen more frequently in the delayed tests.

period. The patient following TV $+P$ was studied two and a quarter years after operation and had a much faster PSP frequency, mean $7.47 \mathrm{c} / \mathrm{m}$ (range 7.0 to $7.9 \mathrm{c} / \mathrm{m}$ ), alternating with periods of inhibition lasting on average 35 seconds (range 16-58 sec). Regular activity with a mean frequency of $3.26 \mathrm{c} / \mathrm{m}$ was present for only $9.2 \%$ of the recording period. When studied 11 months after HSV one other patient also had faster activity alternating with periods of inhibition (fig 6). The frequency of the PSP was $6.44 \mathrm{c} / \mathrm{m}$ (range 4.6 to $7.4 \mathrm{c} / \mathrm{m}$ ) and periods

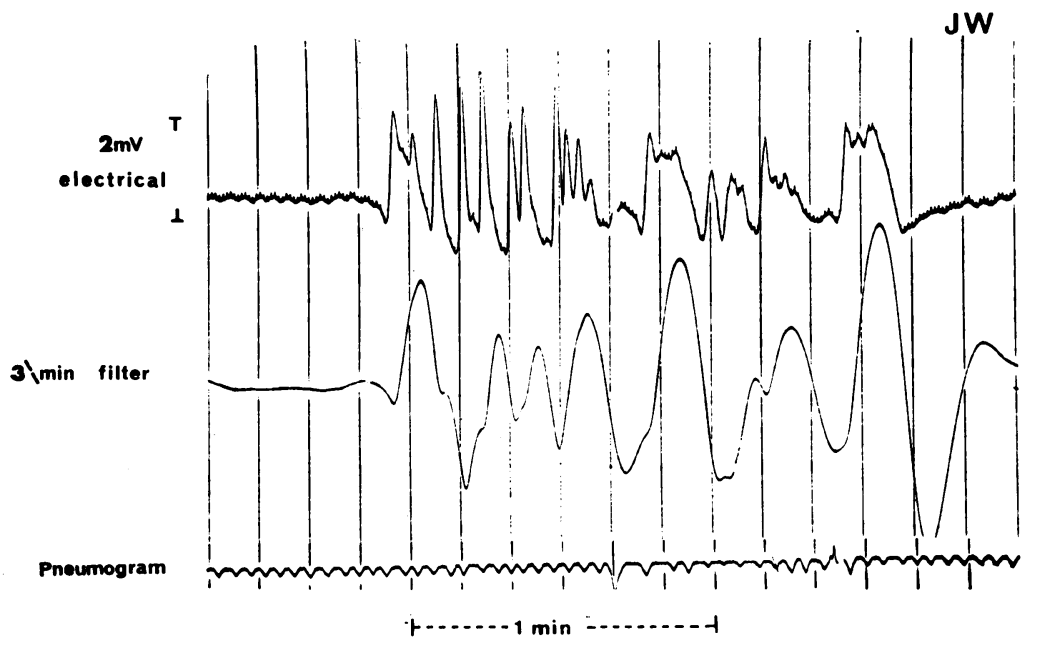

Fig 6 Recording of electrical activity from the gastric antrum of a patient 11 months after highly selective vagotomy. Irregular activity is present and pacesetter potentials of varying wave shape, amplitude, and frequency can be seen alternating with periods of inhibition. 
of inhibition lasted for an average of 57 seconds (range 20-103 sec) with regular activity for only $7.8 \%$ of the recording. The mean amplitude of these irregular electrical waves was 2.28 and $2.22 \mathrm{mV}$ respectively. After the control period each patient received atropine $0.2 \mathrm{mg}$ intravenously as a bolus injection and in each case this was followed by stabilization of the electrical activity. In the patient after $T V+P$ regular activity appeared six and a half minutes after injection and lasted seven and a half minutes (frequency $2.91 \mathrm{c} / \mathrm{m}$ ) and after HSV appeared five minutes after injection and persisted for $14 \mathrm{~min}$ utes. In each patient a further injection of atropine once more stabilized the antral electrical activity.

Short periods of abnormal activity were also present in six other patients studied. One patient after HSV had regular activity during the control period but six minutes after intravenous insulin the frequency of the PSP became much faster (mean frequency $7.6 \mathrm{c} / \mathrm{m}$ ) and this persisted for the rest of the recording. Four patients after TV $+P$ had periods of faster activity present, mean frequency $5.4 \mathrm{c} / \mathrm{m}$ (range 3.9 to $7.3 \mathrm{c} / \mathrm{m}$ ) lasting 70 seconds to three and a half minutes, and one other patient after TV $+P$ had a period of inhibition lasting 45 seconds with no associated faster activity.

\section{MOTOR ACTIVITY}

During the control period motor activity was present for a similar period of time after each type of operation and occurred less frequently in the immediate postoperative tests than in the delayed tests although this difference was not statistically significant. From table III it can be seen that antral motor activity increases in response to insulin hypoglycaemia after HSV but not after TV + P. In one patient with an early positive insulin test after TV $+\mathbf{P}$ at a delayed test, motor activity could not be recorded due to a technical fault.

\begin{tabular}{lllll}
\hline & & $\begin{array}{l}\text { Control } \\
\text { Period }\end{array}$ & \multicolumn{2}{c}{ After Insulin } \\
\cline { 3 - 5 } & & & $0-1$ Hour & $1-2$ Hours \\
\hline Immediate & HSV & 0.2 & 3.5 & 4.0 \\
& TV + P & 0.4 & 0.8 & 1.2 \\
Delayed & HSV & 2.6 & 7.1 & 12.3 \\
& TV + P & 1.7 & 1.6 & 1.8 \\
\hline
\end{tabular}

Table III The percentage time that motor activity was present before and for two hours after intravenous insulin for each type of vagotomy

\section{Discussion}

Antral myoelectrical activity is basically myogenic in origin but can be influenced by both neural and humoral stimuli. We have previously suggested that the changes seen in gastric myoelectrical activity immediately after vagotomy are due to a temporary imbalance between the vagal and sympathetic innervation of the stomach. Since the gastric electrical activity can be likened to a matrix of relaxation oscillators (Sarna, Daniel, and Kingma, 1972) alterations in the degree of coupling may occur following vagotomy and result in the infrequent presence of regular activity in the early postoperative period. A gradual adaptation to this altered autonomic innervation could result in a return of more gradual coupling between oscillators and therefore in the return of regular activity. Some patients in the early postoperative tests had considerable periods of regular activity so adaptation following vagotomy can be expected in the first few weeks after operation. This concept agrees with our findings in this study that the electrical activity of those patients tested between two and three weeks after operation did not differ from patients tested many years postoperatively.

However, complete normality does not return as shown by the persistent decrease in amplitude of the PSP after both types of vagotomy and alteration of waveshape after truncal vagotomy. The amplitude of the PSP is dependent on the change in electrical potential of the extracellular fluid around the cells which are in immediate contact with the recording electrode. A reduced coordination between cells after vagotomy would result in a reduced amplitude of the PSP: the greater the extent of gastric denervation the less the amplitude. The larger proportional reduction in amplitude which we have found would appear to agree with this theory. The antral electrical waves are sinusoidal in shape after TV and resemble those which occur in the more proximal stomach of normal patients (Couturier, Rozé, Paolaggi, and Debray, 1972); this change may also be due to diminished intercellular coordination. Alternatively, El-Sharkawy and Daniel (1973) have suggested that, on an ionic basis, the initial depolarization wave of a triphasic wave may result from an increase in cellular $\mathrm{Na}^{+}$permeability and the 'plateau' phase is maintained by an increase in $\mathrm{Cl}-$ permeability. If vagotomy alters $\mathrm{Na}^{+}$permeability then a sinusoidal wave would result.

In this series of experiments the frequency of the PSP following vagotomy both in the immediate and delayed tests showed no significant difference from the preoperative frequency. This finding differs from that of Barbara, Bortolotti, Vezzadini, Serantoni, Ciani, Miglioli, and Labo (1973) who reported that vagotomy is followed by an initial decrease in frequency in the immediate postoperative period and later by an increase to significantly higher levels than 
in preoperative recordings. A decrease in frequency immediately following HSV or TV $+P$ is not seen in dogs (Kelly and Code, 1969; Stoddard etal,1973b).

The value of the Hollander test in predicting persistent gastric vagal innervation after vagotomy and the likelihood of recurrent duodenal ulceration has been criticized during the last few years. Eight patients in this series had late positive insulin tests and in none of them were the characteristics of the antral myoelectrical activity different from those patients with negative tests. Of the 52 patients studied only one, following TV $+P$, has been shown to have a recurrent duodenal ulcer and has since received further surgery. This patient with an early positive insulin test had a PSP amplitude of $1.85 \mathrm{mV}$ which is significantly greater than other patients after TV + P. Thus recordings of antral myoelectrical activity might be of value in predicting which patients are liable to develop recurrent ulceration.

Irregular activity has previously been reported in dogs following vagotomy (Nelsen et al, 1967; Kelly and Code, 1969) and also in man following the administration of secretin (Kwong et al, 1972) and in one patient with an oesophageal carcinoma involving the vagal network (Nelsen and Kohatsu, 1968). Nelsen considers that the vagus acts as a controlling influence on the normal pacemakers and removal of this influence creates an electrical environment where ectopic foci of activity can exist. In this study ectopic pacemaker activity was seen in eight subjects, this being for the entire control period in two patients. Kwong et al (1972) used pharmacological doses of secretin and it is unlikely that endogenous secretin could account for the changes we have recorded. If sympathetic domination allows ectopic pacemakers to exist in certain conditions then the stabilization of irregular activity following atropine administration may be due to blockade of preganglionic sympathetic fibres and this would account for the effects recorded in these two patients, one following each type of operation. Further studies of the effects of sympathetic blockade on gastric myoelectrical activity are required. From studies of electrical pacing of the canine stomach (Kelly and La Force, 1972) it can be predicted that the maximum driven frequency of the gastric PSP that can be achieved in dogs is approximately 2.5 times greater than the natural frequency. In the patients with faster electrical activity in this study the greatest frequency achieved was $7.9 \mathrm{c} / \mathrm{m}$ which is 2.52 times greater than the mean postvagotomy frequency of $3.14 \mathrm{c} / \mathrm{m}$.
We have shown that some changes in gastric myoelectrical activity are transient, others permanent. From a clinical point of view it may be possible to use recordings of antral myoelectrical activity to detect which patients are liable to recurrent ulceration and to explain some of the changes in gastric emptying following vagotomy.

\section{References}

Barbara, L., Bortolotti, M., Vezzadini, P., Serantoni, C., Ciani, P. A. Miglioli, M., and Labo, G. (1973). Electrical and mechanical activity of the stomach, serum gastrin levels and acid secretion before and after selective proximal vagotomy for duodenal ulcer. Rendic. Gastroent., 5, 17-18.

Couturier, D., Rozé, C., Paolaggi, J., and Debray, C. (1972). Electrical activity of the normal human stomach: a comparative study of recordings obtained from the serosal and mucosal sides. Amer. J. dig. Dis., 17, 969-976.

Dragstedt, L. R., and Owens, F. M., Jr. (1943). Supra-diaphragmatic section of the vagus nerves in treatment of duodenal ulcer. Proc. Soc. exp. Biol. (N.Y.), 53, 152-156.

Duthie, H. L., Kwong, N. K., Brown, B. H., and Whittaker, G. E. (1971). Pacesetter potential of the human gastroduodenal junction. Gut, 12, 250-256.

El-Sharkawy, T. Y., and Daniel, E. E. (1973). The ionic basis of intestinal control potentials (slow waves). Rendic. Gastroent., 5,9 .

Hollander, F. (1948). Laboratory procedures in the study of vagotomy (with particular reference to the insulin test). Gastroenterology, 11, 419-425.

Kelly, K. A., and Code. C. F. (1969). Effect of transthoracic vagotomy on canine gastric electrical activity, Gastroenterology, 57, 51-58.

Kelly, K. A., and La Force, R. C. (1972). Pacing the canine stomach with electrical stimulation. Amer. J. Physiol., 222, 588-594.

Kwong, N. K., Brown, B. H., Whittaker, G. E., and Duthie, H. L. (1970). Electrical activity of the gastric antrum in man. Brit. J. Surg., 57, 913-916.

Kwong, N. K., Brown, B. H., Whittaker, G. E., and Duthie, H. L. (1972). Effects of gastrin, secretin and cholecystokinin-pancreozymin on the electrical activity, motor activity, and acid output of the stomach in man. Scand. J. Gastroent., 7, 161-170.

McIntyre, J. A., Deitel. M., Baida, M., and Jalil, S. (1969). The human electrogastrogram at operation: a preliminary report. Canad. J. Surg., 12, 275-284.

Morton, H. S. (1954). The potentialities of the electrogastrograph. Ann. roy. Coll. Surg., 15, 351-373.

Nagaoka, K. (1968). Electromyographic study on the mechanism of delayed gastric emptying after vagotomy in dogs. Tohoku J. exp. Med., 95, 1-13.

Nelsen, T. S., Eigenbrodt, E. H., Keoshian, L. A. Bunker, C., and Johnson, L. (1967). Alterations in muscular and electrical activity of the stomach following vagotomy. Arch. Surg., 94, 821-835.

Nelsen, T. S., and Kohatsu, S. (1968). Clinical electrogastrography and its relationship to gastric surgery. Amer. J. Surg., 116, 215-222.

Sarna, S. K., Daniel, E. E., and Kingma, Y. J. (1972). Simulation of the electric-control activity of the stomach by an array of relaxation oscillators. Amer. J. dig. Dis., 17, 299-310.

Stoddard, C. J., Brown, B. H., and Duthie, H. L. (1973a). The effects of varying the extent of the vagotomy on the canine gastric and duodenal myoelectrical activity. (Abstr.) Brit. J. Surg., 60, 913.

Stoddard, C. J., Waterfall, W. E., Brown, B. H., and Duthie, H. L. (1973b). The effects of varying the extent of vagotomy on the myoelectrical and motor activity of the stomach. Gut, 14, 657-664.

Storer, E. H., Thornton, T. F., Jr., and Dragstedt, L. R. (1945). Supradiaphragmatic section of the vagus nerves and gastric motility in patients with peptic ulcer. Proc. Soc. exp. Biol. (N.Y.), 59, 141-142. 